\title{
Utilization of imaging for active surveillance in testicular cancer: Is real-world practice concordant with guidelines?
}

Bishal Gyawali ${ }^{1,2,3}$; Rebecca Griffiths ${ }^{4}$; Andrew G. Robinson ${ }^{1,2}$; Matthew D.F. McInnes ${ }^{5,6}$; Philippe L. Bedard ${ }^{7}$; Christopher M. Booth ${ }^{1,2,3,4}$

${ }^{1}$ Division of Cancer Care and Epidemiology, Queen's Cancer Research Institute, Kingston, ON, Canada; ${ }^{2}$ Department of Oncology, Queen's University, Kingston, ON, Canada; ${ }^{3}$ Department of Public Health Sciences, Queen's University, Kingston, ON, Canada; ${ }^{4}$ ICES, Queen's University, Kingston, ON, Canada; ${ }^{5}$ Department of Radiology, University of Ottawa, Ottawa, ON, Canada; ${ }^{6}$ Cinical Epidemiology Program, Ottawa Hospital Research Institute, Ottawa, ON, Canada; ${ }^{7}$ Division of Medical Oncology \& Hematology, Department of Medicine, Princess Margaret Cancer Centre, University of Toronto, Toronto, ON Canada

Acknowledgements: Parts of this material are based on data and information provided by Cancer Care Ontario and CIHI. However, the analysis, conclusions, opinions and statements expressed herein are those of the authors and not necessarily those of Cancer Care Ontario or CIHI. This study was supported by the Institute for Clinical Evaluative Sciences (ICES), which is funded by an annual grant from the Ontario Ministry of Health and LongTerm Care (MOHLTC). Parts of this material are based on data and information compiled and provided by CIHI. The opinions, results and conclusions reported in this paper are those of the authors and are independent from the funding sources and CIHI. No endorsement by ICES or the Ontario MOHLTC is intended or should be inferred. Dr. Booth had full access to all the data in the study and takes responsibility for the integrity of the data and the accuracy of the data analysis.

Cite as: Gyawali B, Griffiths R, Robinson AG, et al. Utilization of imaging for active surveillance in testicular cancer: Is real-world practice concordant with guidelines? Can Urol Assoc J 2021 September 24; Epub ahead of print. http://dx.doi.org/10.5489/cuaj.7246

Published online September 24, 2021

Corresponding author: Dr. Christopher M. Booth, Division of Cancer Care and Epidemiology, Queen’s University Cancer Research Institute, Kingston, ON, Canada; booth@queensu.ca

$* * *$

\section{Abstract}

Introduction: Imaging is an integral component of active surveillance following orchiectomy for stage 1 non-seminoma (NSGCT) and seminoma germ cell tumors. In this population-based study, we describe use of imaging among patients with early-stage testicular cancer and evaluate whether they are concordant with guideline recommendations.

Methods: This is a population-based, retrospective cohort study to describe utilization of imaging among all patients with early-stage testicular cancer treated with active surveillance in 
the Canadian province of Ontario. The Ontario Cancer Registry was linked to electronic records of treatment to identify use of chest and abdomen/pelvis imaging. Data from 2000--2010 were included with followup for up to five years for patients with non-seminoma and 10 years for patients with seminoma. The key outcome of interest was the frequency of imaging at temporal milestones following orchiectomy. Compared to the most contemporaneous guidelines in Ontario, any discordant frequency of imaging was defined as underutilization or overutilization. Substantial under- or overutilization was defined as $>1$ imaging test less/more than what was recommended during a 12-month period.

Results: The study population included 569 patients with NSGCT (median age 28) and 1107 with seminoma (median age 37). Among patients with NSGCT, adherence with body imaging was low in years 1-3 of surveillance (range 26-37\%, predominantly underuse) and higher in years 4-5 (63-67\%, predominantly overuse). Adherence with chest imaging was even lower (range 11-34\% during years 1-5). Among patients with seminoma, adherence with abdominal and chest imaging was relatively stable and comparable throughout the 10-year followup period (range $23-47 \%$ abdomen and $28-47 \%$ chest). Multivariable analysis confirmed that underutilization of imaging was more common in recent years. NSGCT histology was associated with underutilization in years 1-2 but overutilization in years 3-5.

Conclusions: In routine clinical practice, patients with testicular cancer commonly receive imaging discordant to the protocol for active surveillance, with a substantial proportion receiving both under- and overutilization at various times during surveillance followup.

\section{Introduction}

Imaging is a critical component of active surveillance following orchiectomy which is now the preferred treatment for patients with stage 1 non-seminoma (NSGCT) or seminoma germ cell tumours. ${ }^{1,2}$ We have previously reported that active surveillance has been widely adopted in routine practice and is associated with excellent survival outcomes. ${ }^{3}$ Surveillance programs include regular imaging of the lungs and abdomen/pelvis. These programs seek to balance the need for regular imaging to identify early recurrent disease with the risks and harms of imaging over-use. Risks of over-use include: discovery of incidental but clinically insignificant findings, radiation exposure, test-related anxiety and decreased quality of life, and financial costs to society. The risks of radiation exposure and subsequent secondary malignancy are relevant in this setting given high expected long-term survival and young age at diagnosis.

Current guidelines for frequency of imaging during surveillance of stage 1 testicular attempt to balance the competing risks of over- and under-utilization of imaging. Previous study has shown that the mean rates of compliance with chest X-ray and CT scans patients with stage I NSGCT treated in Canadian centres were 78\% and 64\% respectively. ${ }^{4}$ Population-based studies can provide insights into patterns of care and outcomes among patients treated in routine clinical 
practice. ${ }^{5,6}$ In this population-based study, we describe use of imaging among patients with earlystage testicular cancer and evaluate whether practice was concordant with contemporaneous guideline recommendations.

\section{Methods}

\section{Study design and population}

This is a population-based, retrospective cohort study to describe utilization of imaging among all patients with early-stage testicular cancer treated with active surveillance in the Canadian province of Ontario. Ontario has a population of nearly 14 million people and a single payer universal health insurance program. All incident cases age 16 years or above who underwent orchiectomy during 2000-2010 and were managed by active surveillance were included. Patients were defined as being on surveillance if they had no chemotherapy, radiation, or retroperitoneal lymph node dissection within 90 days of orchiectomy. The study was approved by the Research Ethics Board of Queen's University, Kingston, Ontario, Canada. This study was designed, analyzed, and reported in accordance with the Strengthening the Reporting of Observational Studies in Epidemiology (STROBE) statement. ${ }^{7}$

\section{Data sources}

The Ontario Cancer Registry (OCR) is a passive, population-based cancer registry that captures diagnostic and demographic information for at least $98 \%$ of all incident cases of cancer in the province of Ontario. ${ }^{8}$ A variety of electronic administrative health databases were linked to the OCR, including ALR (Activity Level Reporting Data), a database of patient level activity on radiation and systemic therapy services for cancer treatment; CIHI (Canadian Institute of Health Information) which contains information on hospitalizations and surgical procedures; and OHIP (Ontario Health Insurance Plan), a database of physician claims for medical services provided. These services include laboratory tests, consults, surgeries, diagnostic tests, and therapeutic procedures.

We obtained surgical pathology reports for all orchiectomy procedures performed in the province of Ontario from 2000-2010. The data were manually abstracted by trained personnel into a pre-piloted electronic database and linked using unique, encoded identifiers to several administrative health databases housed at the Institute for Clinical Evaluation Services. The CIHI database provides information about orchiectomy procedures. OHIP provincial physician billing records, along with electronic treatment records from regional cancer centres, were used to identify chemotherapy utilization. Use of imaging tests was identified from OHIP physician billing records. Data sets were linked with unique encoded identifiers and were analyzed at the Institute for Clinical Evaluation Services. Data from 2000-2010 were included with follow up for up to 5 years for patients with non-seminoma and 10 years for patients with seminoma as per the practice guideline recommendations for duration of follow-up. 


\section{Measures and outcomes}

The key outcome of interest was the frequency of imaging modalities at pre-identified temporal milestones following orchiectomy. Imaging utilization was compared to the guidelines from the Princess Margaret Cancer Centre (Toronto, Ontario) which were the most contemporaneous guidelines in Ontario at the time (1999-2010 for non-seminoma and 1995-2004 for seminoma, Table 1). These guidelines are very similar to guidelines from NCCN and ESMO during the study period (no public links available for these past versions of the guidelines, authors can provide them upon request).

Under-utilization and over-utilization were defined for each year for CT abdomen/pelvis (CT AP) and chest imaging (CT Chest or CXR) separately and were defined as imaging frequencies less than or more than the frequencies listed in Table 1. Year of imaging was measured from date of orchiectomy; imaging studies performed in the 2 months prior to orchiectomy were counted as Year 1. Patients were excluded from the denominator for the ongoing and subsequent years in the event of death, further treatment, or incomplete availability of imaging data. A difference in the frequency by more than 1 were considered substantial overuse ( $>1$ over) or substantial underuse $(<1$ under).

\section{Statistical analysis}

We report the proportion of patients with over/under-utilization of imaging. Univariate and multivariate logistic regression was used to identify factors associated with over/underutilization. Patients with over/under-utilization in any of the years of follow-up were classified as over/under-utilization "yes". Co-variates considered in the model included year of orchiectomy, age group, region, tumor type (seminoma or non-seminoma) and high-risk (defined as lymphovascular invasion positive for non-seminoma and tumor $>4 \mathrm{~cm}$ or rete testes invasion for seminoma risk) or low-risk for relapse. These analyses were done for years 1 and 2 and years 3-5 separately because the recommended frequencies of imaging drop substantially between first 2 years and rest of the years. For the regression analysis, we only included 5 years of follow-up for seminoma patients to be consistent with non-seminoma (i.e. years 6-10 were not considered) since both histologies were included in the model. Any patients who did not have complete follow-up were excluded from analysis for that year and subsequent years. Any cohort with 5 or fewer number of patients were not considered in the analyses. All the analyses were conducted in SAS version 9.4

\section{Results}

\section{Study population}

During 2000-2010, 3546 patients were diagnosed with testicular cancer in Ontario, including 3281 who had an orchiectomy identified (93\%) (Supplemental eFigure 1). Pathology reports were available for $2821(86 \%)$ of these cases. There were no significant differences in demographics, histology, or survival of those cases with $(n=2821)$ and without $(n=460)$ available 
orchiectomy pathology reports (Supplemental eFigure 2 and eTable 1). One hundred (4\%) cases were excluded as the histology was not germ-cell tumour, $36(1 \%)$ cases were excluded as the date of pathology report and orchiectomy were not consistent, $35(1 \%)$ cases were excluded as they has other cancer-directed therapy before orchiectomy, and 974 patients were excluded who had radiation or systemic treatment within 90 days of orchiectomy. The study population included 1676 patients; 569 with NSGCT and 1107 with seminoma. Characteristics of the study population are show in Table 1. Median age was 34 (28 for NSGCT and 37 for seminoma). Twenty-six percent (149/569) of NSGCT and 35\% (584/1107) of seminoma patients were classified as having high-risk disease.

\section{Utilization of imaging}

The pattern of under-imaging and over-imaging for both seminoma and non-seminoma cohort are provided in Figure 1.

\section{Non-seminoma}

As shown in Table 3, among patients with NSGCT, adherence with body imaging was low in Years 1-3 of surveillance (range 26-37\%) and higher in Years 4-5 (63-67\%). In the earlier years, under-utilization of imaging was more common; this pattern shifted to over-utilization in later years. Substantial under-use of body imaging (i.e. $>1$ under-reported scan per year) was observed in $20 \%(87 / 432)$ and $36 \%(148 / 414)$ of patients in Years 1 and 2 . Substantial over-use of body imaging (i.e. $>1$ additional scan per year) was highest in Year $3(28 \%, 104 / 368)$.

Adherence with chest imaging was even lower (range 11 to 34\% during Years 1-5). Substantial under-use of chest imaging (i.e. $>1$ under-reported scan per year) was highest ins Year 1 and 2 (57\% and 73\% respectively); substantial over-use of chest imaging (i.e. $>1$ additional scan per year) was relatively uncommon (range 1-12\% in Years 1-5).

\section{Seminoma}

As shown in Table 3, among patients with seminoma, adherence with abdominal and chest imaging was relatively stable and comparable throughout the 10-year follow-up period (range $23-47 \%$ abdomen and $28-47 \%$ chest). A substantial number of patients had under-utilization of abdominal imaging for seminoma (range 48-77\%) with substantial under-utilization in 21-42\%. There was very little over-use of abdominal imaging for seminoma. Chest imaging was more likely to be under- and over-utilized. Rates of under-use increased over time (from 14\% in Year 1 to $58 \%$ in Year 10); over-utilization rates decreased over time (57\% in Year 1 to $10 \%$ in Year $10)$.

\section{Factors associated with under/over-utilization of imaging}

Under-utilization of imaging was more common in later years (OR per year 1.14, 95\%CI 1.061.23) and among those patients with NSGCT (OR 1.87, 95\%CI 1.08-3.24) during Years 1 and 2 (Table 4). These associations persisted in multivariate analysis (adjusted OR for years 1.15 (1.06-1.25) and for NSGCT histology 2.11 (1.01-4.38)). Patients with orchiectomy in later years 
were more likely to have underutilization for years 3-5 as well [adjusted OR 1.09 (1.01-1.19)]. Compared with seminoma, patients with NSGCT were less likely to have underutilization during years 3-5 of follow-up [adjusted OR, $0.53(0.34-0.84)]$.

Over-utilization of imaging in Years 1-2 was more common among patients with NSGCT [adjusted OR $0.24(0.18-0.33)$ ]. During years 3-5, NSGCT was associated with higher odds of overutilization [adjusted OR for NSGCT 4.01 (2.58-6.24)]. For years 3-5, although low-risk disease was associated with overutilization in the univariate analysis (OR 1.67(1.23-2.27)), this did not persist when adjusted in the multivariable analysis.

There was substantial regional variation in over-utilization (range 50-100\%) but not under-utilization (range 80-88\%). In multivariable analysis, over-utilization was but underutilization was not associated with geographical region both for years 1-2 and years 3-5.

\section{Discussion}

In this population-based study we have described use of imaging among patients with testicular cancer managed by active surveillance. The study has highlighted substantial discordance with imaging guidelines. Our data show substantial rates of under-utilization and over-utilization that vary during the different phases of surveillance follow-up.

Cancer treatment must balance efficacy and toxicity. Treatment of testicular cancer is one of the success stories in oncology in which high rates of success have been achieved; recent focus in this disease has been to minimize the toxicity of therapy. High rates of cures are achievable in testicular cancer, but numerous de-escalation trials have proven that high cure rates could be maintained with less intense treatment. This is particularly true for stage I testicular cancer in which up-front adjuvant RT (for seminoma) or RPLND/adjuvant chemotherapy (for NSGCT) has been largely replaced by active surveillance. This practice was driven by the data which showed excellent outcomes can be achieved with less intensive therapy while simultaneously reducing treatment-related toxicity. ${ }^{9}$ Active surveillance is also shown to be the most cost-effective modality of treatment. ${ }^{10}$ Our previous study using the same database has shown that active surveillance is now the most common treatment strategy for patients with stage I testicular cancer in Ontario. ${ }^{3}$

However, active surveillance requires structured follow-up to maintain its effectiveness. ${ }^{11}$ Surveillance is able to achieve excellent survival in large part due to regular and frequent monitoring with imaging and serum tumour markers to detect early relapse. Indeed, up to 15$50 \%$ of patients with stage I non-seminoma, and $10-20 \%$ of patients with seminoma relapse after AS. ${ }^{12}$ Therefore, cancer care guidelines, such as the NCCN, ESMO or the CCO recommend chest and abdomen/pelvis imaging at various frequencies and intervals for up to 5 (nonseminoma) or 10 years (seminoma) post-orchiectomy.

Our study finds that in routine practice, patients with testicular cancer commonly receive imaging tests at a rate that is discordant with recommended guidelines. This finding raises several interesting questions and hypotheses. First, it shows the complexities of treating patients with cancer in the real-world setting. Delivery of guideline-based care for testicular cancer is 
very important because most patients (even with advanced disease) can be cured. However, our study identifies important gaps with care delivery in routine practice. We found that rates of discordance (and whether it was under or over-utilization) were largely related to the number of years from orchiectomy. This likely reflects changes in clinical practice (i.e. clinicians might be more or less likely to order tests) as well as the fact that guidelines have different levels of imaging intensity during the years of follow-up. We also observed significant variation across regions, specially with regards to overutilization which is not surprising as practice patterns in this context will vary from centre to centre.

Second, the guidelines for frequency of imaging during active surveillance for testicular cancers are based on consensus, informed by data regarding the timing of relapse

following orchidectomy. That may also explain why the physicians are not rigorous in following the guideline recommendations with regards to surveillance protocol for imaging; it may also reflect the fact that the disease remains highly curable even when advanced. There are also patient-related factors that may explain the lack of adherence to surveillance guidelines. The patient population for testicular cancers is unique in that the cohort comprises mostly of young men who are working, mobile and may not routinely adhere to the surveillance protocol.

Third, our study suggests that overuse of imaging is very common particularly for chest imaging in the early years of seminoma and body imaging in the later years for NSGCT. Overuse of imaging has its downstream hazards including excessive exposure to radiation and detection of indeterminate lesions leading to downstream invasive diagnostic and therapeutic procedures. Over-imaging and all these downstream cascades of medical interventions also incur substantial costs to the payer.

An important question that arise from our study is whether underuse of imaging during surveillance leads to inferior outcomes. While existing data-sets and methodologic limitations do not allow for a definitive answer to this question, it is notable that despite substantial imaging under-use, the outcomes of patients treated with surveillance in Ontario is excellent. A seminal clinical trial report published in 2007 suggested that two CT scans, as opposed to five, did not compromise outcomes in patients with low-risk stage I non-seminoma germ cell tumors. ${ }^{13} \mathrm{New}$ data from the TRISST trial also shows non-inferiority of 3 scans during surveillance (at months 6,18 and 36) compared with 7 scans (at months 6,12,18,24,36,48,60) among patients with stage I seminoma and suggests imaging maybe unnecessary beyond 3 years. ${ }^{14}$ Despite the detection of under-use and over-use of imaging in our study, our previous study using the same cohort of patients as this study revealed a 5 year survival rate of $97 \%$ and cancer specific survival of $98 \%$ that is consistent with global data. ${ }^{3}$ However, our data do not support purposeful delivery of care that is not consistent with guidelines. Future studies should focus on further reducing unnecessary imaging to this population.

Our study should be interpreted in light of methodologic limitations. Existing datasets do not include stage of disease or serum tumour markers. Accordingly, we defined patients on surveillance as those patients treated with orchiectomy and no further RT/chemotherpay/RPLND 
within the subsequent 90 days. Lack of information regarding tumour markers and indication for imaging means that we do not know if radiographic investigations were performed in response to new symptoms (or rising tumour markers), follow-up of prior equivocal findings, or were planned as per routine surveillance; this means that some apparent over-use may in fact be appropriate. One way to address this would have been to censor patients a certain number of days or at the penultimate scans before they re-started any treatment, but there is no agreement on the ideal number of days and these approaches are also at risk of falsely underreporting surveillance scans. We have also not reported imaging use beyond the usual surveillance periods ( 5 years for NSGCT and 10 years for seminoma); accordingly we may under-estimate over-use of imaging as some patients may continue to have routine tests beyond the recommended time frames. In order to measure the extent to which practice was concordant with contemporaneous guidelines, we compared imaging frequency to guidelines from an earlier era which were published by Ontario's largest cancer centre. While many oncologists in Ontario used these guidelines at that time, this may limit the extent to which these findings apply to the current era. Finally, our study design does not take into account patients that were lost to follow-up entirely and for whom we have no further information about imaging after Year 1.

In summary, this study illustrates that use of surveillance imaging among patients with early stage testicular cancer is frequently discordant with guideline recommendations. We have observed substantial under- and over-utilization. Future clinical trials should evaluate whether imaging protocols can be safely de-escalated and how to reduce unnecessary over-utilization of imaging.

Conflicts of interest: Dr. Gyawali reports receiving consulting fees from Vivio Health. Dr. Bedard reports receiving consulting fees from Seattle Genetics, Lilly, Amgen, Merck, BMS and Pfizer, and research funding for his institution from BMS, Sanofi, Astrazeneca, Genetech/Roche, Servier, GSK, Novartis, PTC Therapeutics, Nektar, Merck, Seattle Genetics, Mersana, Immunomedics, Lilly, Amgen and Bicara. Dr. Robinson reports receiving honoraria from Merck, consulting fees from Merck, Astrazeneca, and Amgen and research funding from Astrazeneca, Merck, BMS and Roche. No disclosures to report for other authors. 


\section{References}

1. Gilligan T, Lin DW, Aggarwal R, et al. Testicular Cancer, Version 2.2020, NCCN Clinical Practice Guidelines in Oncology. 2019;17(12):1529.

2. Oldenburg J, Fosså SD, Nuver J, et al. Testicular seminoma and non-seminoma: ESMO Clinical Practice Guidelines for diagnosis, treatment and follow-up $\dagger$. Annals of Oncology. 2013;24:vi125-vi132.

3. Leveridge MJ, Siemens DR, Brennan K, et al. Temporal trends in management and outcomes of testicular cancer: A population-based study. Cancer. 2018;124(13):27242732.

4. Ernst DS, Brasher P, Venner PM, et al. Compliance and outcome of patients with stage 1 non-seminomatous germ cell tumors (NSGCT) managed with surveillance programs in seven Canadian centres. Can J Urol. 2005;12(2):2575-2580.

5. Booth CM, Mackillop WJ. Translating new medical therapies into societal benefit: the role of population-based outcome studies. Jama. 2008;300(18):2177-2179.

6. Booth CM, Karim S, Mackillop WJ. Real-world data: towards achieving the achievable in cancer care. Nat Rev Clin Oncol. 2019;16(5):312-325.

7. von Elm E, Altman DG, Egger M, et al. The Strengthening the Reporting of Observational Studies in Epidemiology (STROBE) Statement: guidelines for reporting observational studies. Int J Surg. 2014;12(12):1495-1499.

8. Clarke EA, Marrett LD, Kreiger N. Cancer registration in Ontario: a computer approach. IARC Sci Publ. 1991(95):246-257.

9. Pierorazio PM, Cheaib JG, Patel HD, et al. Comparative Effectiveness of Surveillance, Primary Chemotherapy, Radiotherapy, and Retroperitoneal Lymph Node Dissection for the Management of Early-Stage Testicular Germ Cell Tumors: A Systematic Review. J Urol. 2020:101097ju0000000000001364.

10. Huang MM, Su ZT, Cheaib JG, et al. Cost-effectiveness Analysis of Non\&\#x2013;riskadapted Active Surveillance for Postorchiectomy Management of Clinical Stage I Seminoma. European Urology Focus.

11. Kollmannsberger C, Tyldesley S, Moore C, et al. Evolution in management of testicular seminoma: population-based outcomes with selective utilization of active therapies. Annals of oncology : official journal of the European Society for Medical Oncology. 2011;22(4):808-814.

12. Nappi L, Nichols CR, Kollmannsberger CK. New treatments for stage I testicular cancer. Clin Adv Hematol Oncol. 2017;15(8):626-631.

13. Rustin GJ, Mead GM, Stenning SP, et al. Randomized trial of two or five computed tomography scans in the surveillance of patients with stage I nonseminomatous germ cell tumors of the testis: Medical Research Council Trial TE08, ISRCTN56475197--the National Cancer Research Institute Testis Cancer Clinical Studies Group. J Clin Oncol. 2007;25(11):1310-1315.

14. Joffe JK, Cafferty FH, Murphy L, et al. Imaging modality and frequency in surveillance of stage I seminoma testicular cancer: Results from a randomized, phase III, factorial trial (TRISST). Journal of Clinical Oncology. 2021;39(6_suppl):374-374. 


\section{Figures and Tables}

Fig. 1. Pattern of under-imaging and over-imaging for both seminoma and non-seminoma cohort. (A) Seminoma overuse. * Suppressed owing to small cell count

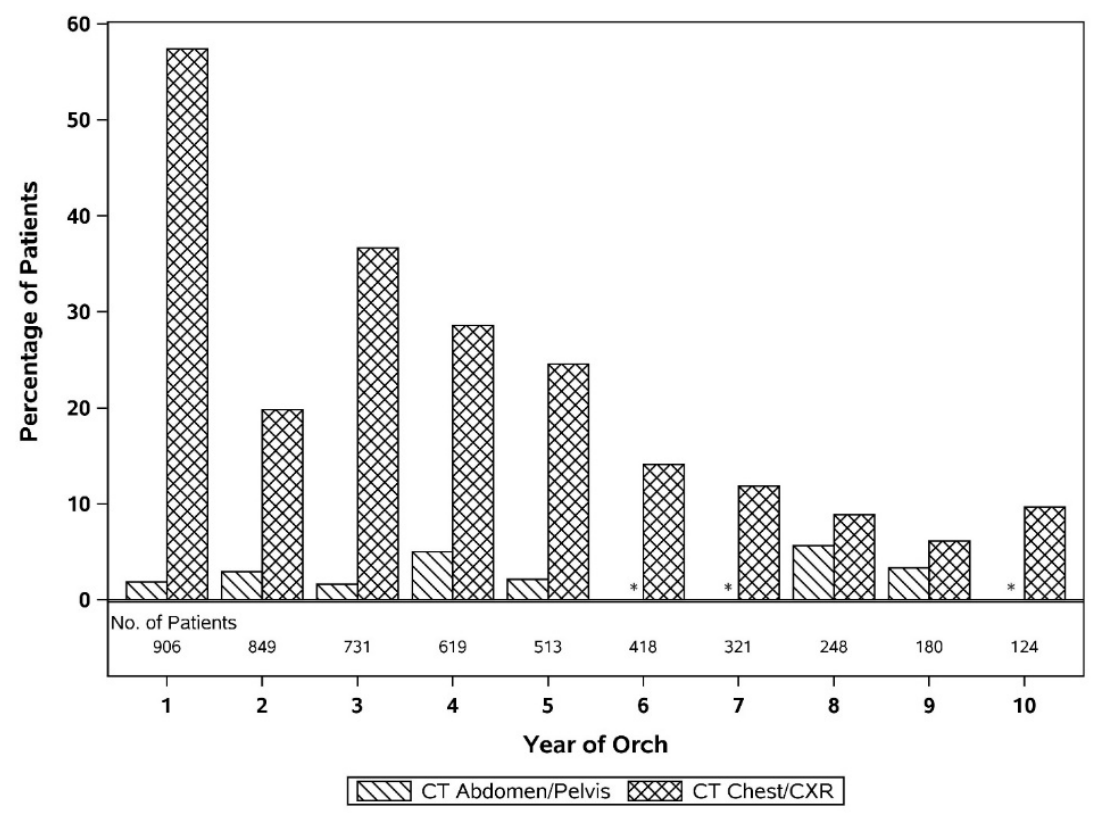

(B) Seminoma underuse.

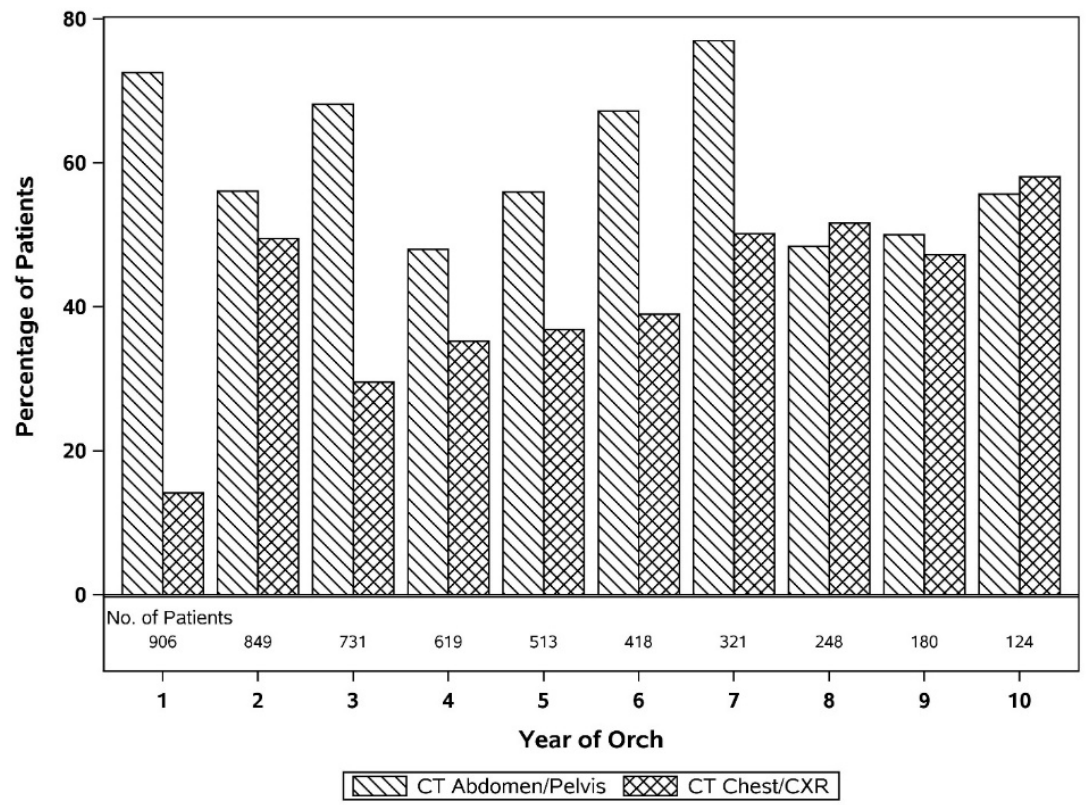


(C) Non-seminoma overuse.

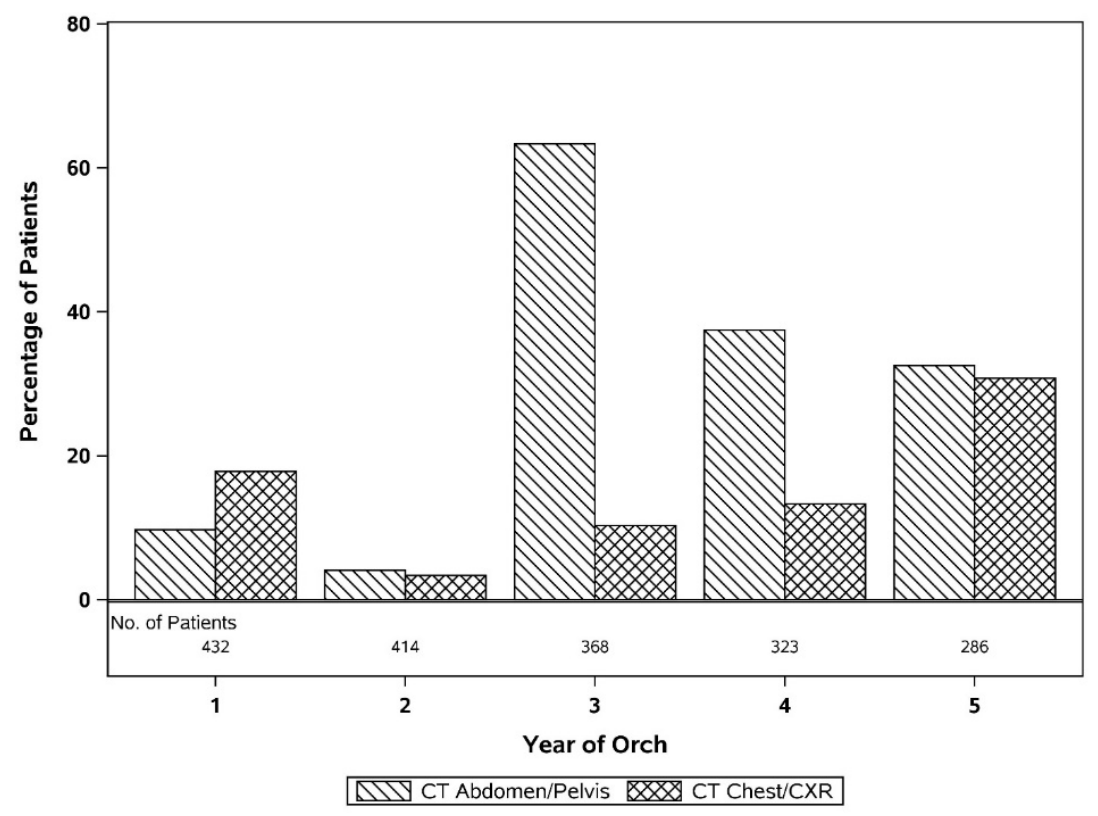

(D) Non-seminoma underuse.

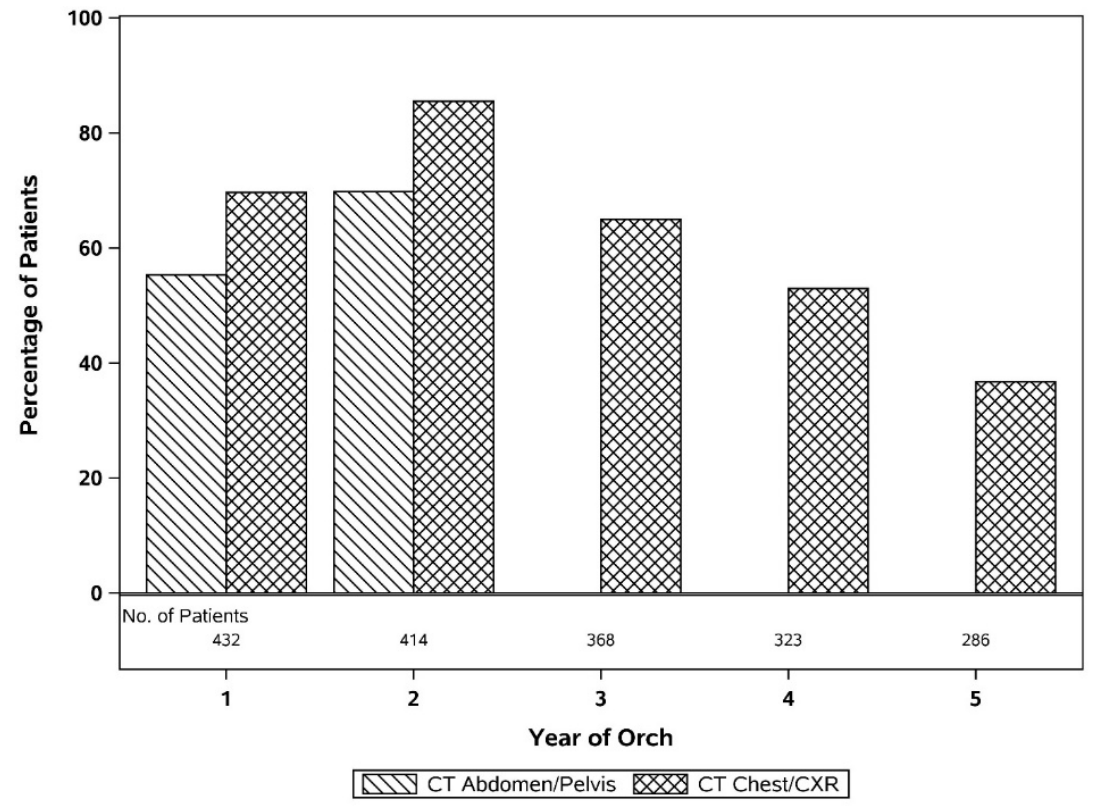




\begin{tabular}{|c|c|c|c|c|}
\hline \multirow[t]{2}{*}{ Year } & \multicolumn{2}{|c|}{ Non-seminoma annual tests } & \multicolumn{2}{|c|}{ Seminoma annual tests } \\
\hline & CT abdomen/pelvis & CT chest or CXR & CT abdomen/pelvis & $\begin{array}{c}\text { CT chest or } \\
\text { CXR }\end{array}$ \\
\hline 1 & 4 & 7 & 4 & 2 \\
\hline 2 & 3 & 6 & 3 & 2 \\
\hline 3 & 0 & 3 & 3 & 1 \\
\hline 4 & 0 & 2 & 2 & 1 \\
\hline 5 & 0 & 1 & 2 & 1 \\
\hline 6 & NA & NA & 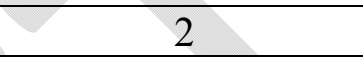 & 1 \\
\hline 7 & NA & NA & 2 & 1 \\
\hline 8 & $\mathrm{NA}$ & $\mathrm{NA}$ & 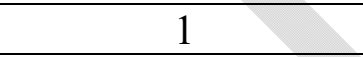 & 1 \\
\hline 9 & $\mathrm{NA}$ & NA & 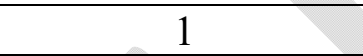 & 1 \\
\hline 10 & NA & NA & 1 & 1 \\
\hline
\end{tabular}

Year is measured from discharge date of orchiectomy. Year 1 includes imaging done 2 months before orchiectomy. CT: computed tomography; CXR: chest X-ray; NA: not applicable.

\begin{tabular}{|c|c|c|c|}
\hline Characteristic & All patients & Non-seminoma & seminoma \\
\hline & $N=1676$ & $N=569$ & $\mathrm{~N}=1107$ \\
\hline \multicolumn{4}{|c|}{ n (\%) } \\
\hline 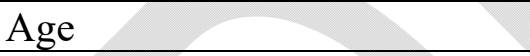 & 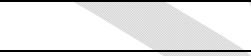 & & \\
\hline Mean/Median (years) & $36 / 34$ & $31 / 28$ & $38 / 37$ \\
\hline$<20$ & $84(5.0 \%)$ & $64(11 \%)$ & $20(2 \%)$ \\
\hline $20-29$ & $466(28 \%)$ & $246(43 \%)$ & $220(20 \%)$ \\
\hline $30-39$ & $570(34 \%)$ & $160(28 \%)$ & $410(37 \%)$ \\
\hline $40-49$ & $385(230 \%)$ & $75(13 \%)$ & $310(28 \%)$ \\
\hline $50-59$ & $122(7 \%)$ & $18(3 \%)$ & $104(9 \%)$ \\
\hline $60+$ & $49(3 \%)$ & $6(1 \%)$ & $43(4 \%)$ \\
\hline \multicolumn{4}{|l|}{ Primary histology } \\
\hline Seminoma, NOS & $1207(72.0 \%)$ & $100(17.6 \%)$ & $1107(100.0 \%)$ \\
\hline Embryonal carcinoma, NOS & $267(15.9 \%)$ & $267(46.9 \%)$ & $\mathrm{NA}$ \\
\hline Teratoma, malignant, NOS & $147(8.8 \%)$ & $147(25.8 \%)$ & NA \\
\hline Yolk sac tumor & $51(3.0 \%)$ & $51(9.0 \%)$ & NA \\
\hline Choriocarcinoma, NOS & $\leq 5$ & $\leq 5$ & NA \\
\hline NA & $\leq 5$ & $\leq 5$ & NA \\
\hline \multicolumn{4}{|l|}{ Tumor size $(\mathrm{cm})$} \\
\hline Mean/median & $4 / 4$ & $4 / 4$ & $4 / 4$ \\
\hline
\end{tabular}




\begin{tabular}{|l|c|c|c|}
\hline$\leq 4 \mathrm{~cm}$ & $979(58 \%)$ & $326(57 \%)$ & $653(59 \%)$ \\
\hline$>4 \mathrm{~cm}$ & $655(40 \%)$ & $233(41 \%)$ & $422(38 \%)$ \\
\hline Unstated & $42(3 \%)$ & $10(2 \%)$ & $32(3 \%)$ \\
\hline Rete testis invasion & & & \\
\hline Yes & $440(26 \%)$ & $127(22 \%)$ & $313(28 \%)$ \\
\hline No & $677(40 \%)$ & $219(38 \%)$ & $458(41 \%)$ \\
\hline Not stated & $559(33 \%)$ & $223(39 \%)$ & $336(30 \%)$ \\
\hline Lymphovascular invasion & & & \\
\hline Yes & $306(18 \%)$ & $149(26 \%)$ & $157(14 \%)$ \\
\hline No & $1,023(61 \%)$ & $298(52 \%)$ & $725(65 \%)$ \\
\hline Not stated & $347(21 \%)$ & $122(21 \%)$ & $225(20 \%)$ \\
\hline
\end{tabular}

$\%$ might not add to $100 \%$ due to rounding. NA: not applicable; NOS: not otherwise specified.

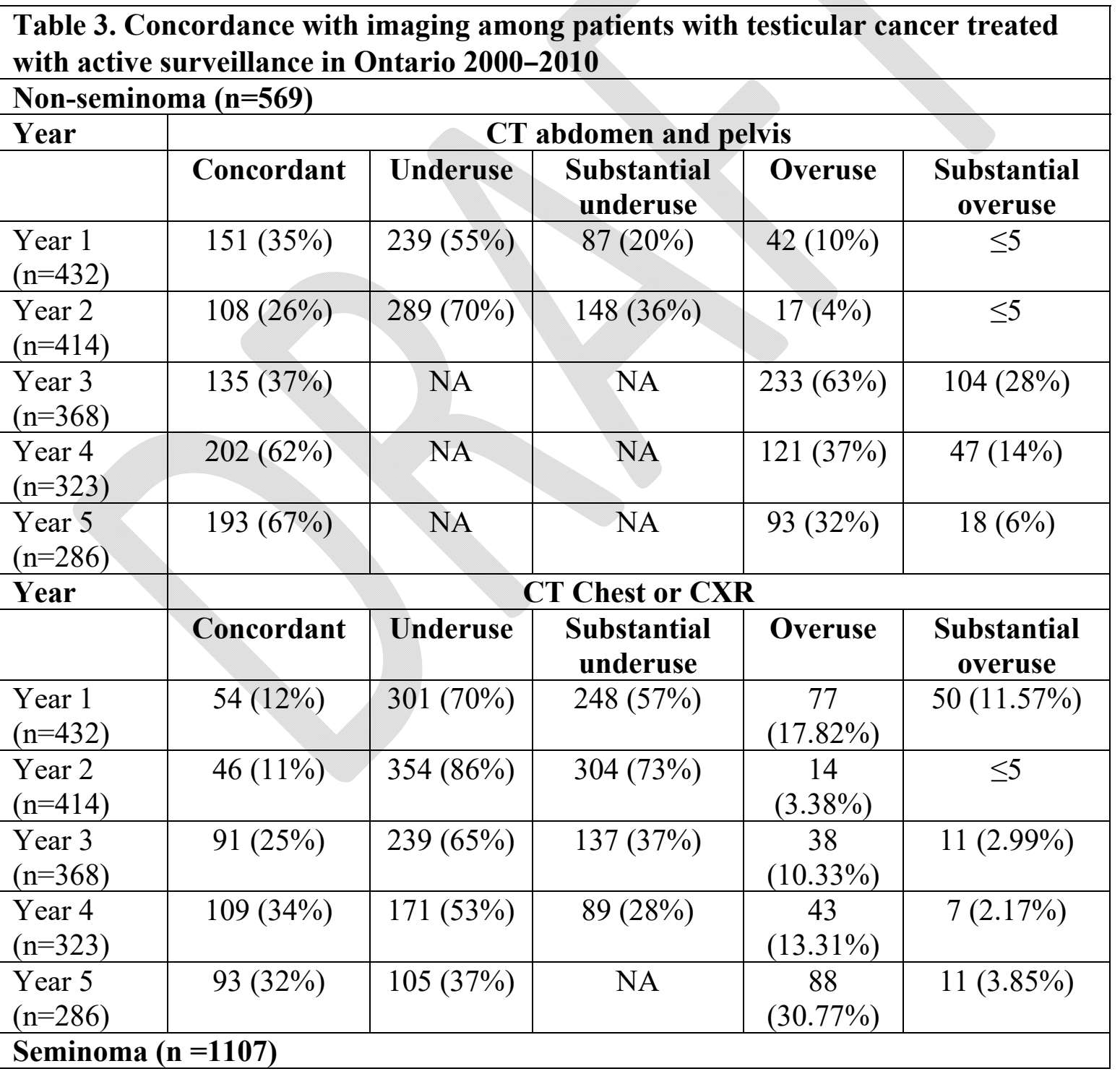




\begin{tabular}{|c|c|c|c|c|c|}
\hline \multirow[t]{2}{*}{ Year } & \multicolumn{5}{|c|}{ CT abdomen and pelvis } \\
\hline & Concordant & Underuse & $\begin{array}{c}\text { Substantial } \\
\text { underuse }\end{array}$ & Over-use & $\begin{array}{c}\text { Substantial } \\
\text { overuse }\end{array}$ \\
\hline $\begin{array}{l}\text { Year } 1 \\
(n=906)\end{array}$ & $232(26 \%)$ & $657(73 \%)$ & $194(21 \%)$ & $17(2 \%)$ & $\leq 5$ \\
\hline $\begin{array}{l}\text { Year } 2 \\
(\mathrm{n}=849)\end{array}$ & $348(41 \%)$ & $476(56 \%)$ & $212(25 \%)$ & $25(3 \%)$ & $\leq 5$ \\
\hline $\begin{array}{l}\text { Year } 3 \\
(n=731)\end{array}$ & $221(30 \%)$ & $498(68 \%)$ & $272(37 \%)$ & $12(2 \%)$ & $\leq 5$ \\
\hline $\begin{array}{l}\text { Year } 4 \\
(n=619)\end{array}$ & $291(47 \%)$ & $297(48 \%)$ & $152(24)$ & $31(5 \%)$ & $\leq 5$ \\
\hline $\begin{array}{l}\text { Year } 5 \\
(n=513)\end{array}$ & $215(42 \%)$ & $287(56 \%)$ & $154(30 \%)$ & $11(2 \%)$ & $\leq 5$ \\
\hline $\begin{array}{l}\text { Year } 6 \\
(\mathrm{n}=418)\end{array}$ & $132(32 \%)$ & $281-285$ & $135(32 \%)$ & $\leq 5$ & $\leq 5$ \\
\hline $\begin{array}{l}\text { Year } 7 \\
(\mathrm{n}=321)\end{array}$ & $73(23 \%)$ & $243-247$ & $136(42 \%)$ & $\leq 5$ & $\leq 5$ \\
\hline $\begin{array}{l}\text { Year } 8 \\
(\mathrm{n}=248)\end{array}$ & $114(46 \%)$ & $120(48 \%)$ & NA & $14(6 \%)$ & $\leq 5$ \\
\hline $\begin{array}{l}\text { Year 9 } \\
(\mathrm{n}=180)\end{array}$ & $84(47 \%)$ & $90(50 \%)$ & NA & $6(3 \%)$ & $\leq 5$ \\
\hline $\begin{array}{l}\text { Year 10 } \\
(\mathrm{n}=124)\end{array}$ & $51(41 \%)$ & $68-73$ & NA & $\leq 5$ & $\leq 5$ \\
\hline Year & & & T chest or CI & & \\
\hline & Concordant & Underuse & $\begin{array}{c}\text { Substantial } \\
\text { underuse }\end{array}$ & Over-use & $\begin{array}{c}\text { Substantial } \\
\text { overuse }\end{array}$ \\
\hline $\begin{array}{l}\text { Year 1 } \\
(n=906)\end{array}$ & $258(28 \%)$ & $128(14 \%)$ & $15(2 \%)$ & $520(57 \%)$ & $273(30 \%)$ \\
\hline $\begin{array}{l}\text { Year } 2 \\
(n=849)\end{array}$ & $261(31 \%)$ & $420(49 \%)$ & $167(20 \%)$ & $168(20 \%)$ & $63(7 \%)$ \\
\hline $\begin{array}{l}\text { Year } 3 \\
(n=731)\end{array}$ & $247(34 \%)$ & $216(29 \%)$ & NA & $268(37 \%)$ & $103(14 \%)$ \\
\hline $\begin{array}{l}\text { Year } 4 \\
(n=619)\end{array}$ & $224(36 \%)$ & $218(35 \%)$ & NA & $177(28 \%)$ & $46(7 \%)$ \\
\hline $\begin{array}{l}\text { Year } 5 \\
(n=513)\end{array}$ & $198(39 \%)$ & $189(37 \%)$ & NA & $126(24 \%)$ & $26(5 \%)$ \\
\hline $\begin{array}{l}\text { Year } 6 \\
(n=418)\end{array}$ & $196(47 \%)$ & $163(39 \%)$ & NA & $59(14 \%)$ & $10(2 \%)$ \\
\hline $\begin{array}{l}\text { Year } 7 \\
(n=321)\end{array}$ & $122(38 \%)$ & $161(50 \%)$ & NA & $38(12 \%)$ & $8(2 \%)$ \\
\hline $\begin{array}{l}\text { Year } 8 \\
(n=248)\end{array}$ & $98(39 \%)$ & $128(52 \%)$ & NA & $22(9 \%)$ & $\leq 5$ \\
\hline $\begin{array}{l}\text { Year } 9 \\
(n=180)\end{array}$ & $84(47 \%)$ & $85(47 \%)$ & NA & $11(6 \%)$ & $\leq 5$ \\
\hline
\end{tabular}




\begin{tabular}{|l|l|l|l|l|l|}
\hline $\begin{array}{l}\text { Year } 10 \\
(\mathrm{n}=124)\end{array}$ & $40(32 \%)$ & $72(58 \%)$ & NA & $12(10 \%)$ & $\leq 5$ \\
\hline
\end{tabular}

CT: computed tomography; CXR: chest X-ray; NA: not applicable.

Table 4. Factors associated with underutilization of surveillance imaging among patients with early-stage testicular cancer in Ontario treated during 2000-2010

\begin{tabular}{|c|c|c|c|c|c|c|}
\hline & \multicolumn{3}{|c|}{ Years 1-2 } & \multicolumn{3}{|c|}{ Years 3-5 } \\
\hline & \multicolumn{2}{|c|}{ Univariate analysis } & \multirow{2}{*}{$\begin{array}{c}\begin{array}{c}\text { Multivariate } \\
\text { analysis }\end{array} \\
\text { OR (95\% CI) } \\
\end{array}$} & \multicolumn{2}{|c|}{ Univariate analysis } & \multirow{2}{*}{$\begin{array}{c}\text { Multivariate analysis } \\
\text { OR }(95 \% \text { CI }) \\
\end{array}$} \\
\hline & Rate & OR $(95 \%$ CI $)$ & & Rate & OR (95\% CI) & \\
\hline $2000-2010^{\wedge}$ & & $1.14(1.06-1.23)$ & $1.15(1.06-1.25)$ & & $1.07(0.99-1.16)$ & $1.09(1.01-1.19)$ \\
\hline \multicolumn{7}{|l|}{ Age } \\
\hline$<30$ years & 96 & Ref & Ref & 84 & Ref & Ref \\
\hline $30-39$ years & 93 & $0.59(0.33-1.07)$ & $0.75(0.39-1.46)$ & 79 & $0.74(0.48-1.13)$ & $0.61(0.37-0.99)$ \\
\hline $40+$ years & 92 & $0.56(0.31-1)$ & $0.63(0.33-1.22)$ & 82 & $0.89(0.57-1.39)$ & $0.69(0.41-1.17)$ \\
\hline \multicolumn{7}{|l|}{ Histology } \\
\hline Non-sem & 96 & $1.87(1.08-3.24)$ & $2.11(1.01-4.38)$ & 77 & $0.61(0.43-0.88)$ & $0.53(0.34-0.84)$ \\
\hline Seminoma & 93 & Ref & Ref & 84 & Ref & Ref \\
\hline \multicolumn{7}{|l|}{ Risk $^{*}$} \\
\hline High & 93 & Ref & Ref & 84 & Ref & Ref \\
\hline Low & 94 & $1.29(0.8-2.08)$ & $1.14(0.7-1.86)$ & 81 & $0.81(0.54-1.19)$ & $0.9(0.59-1.35)$ \\
\hline
\end{tabular}

${ }^{\wedge}$ Per year. ${ }^{*}$ High-risk disease is defined as: LVI for NSGCT; tumor $>4 \mathrm{~cm}$ and/or rete testis involvement for seminoma. Geographic region was included in the multivariate model but was not associated with imaging utilization. CI: confidence interval; OR: odds ratio. 


\section{Table 5. Factors associated with overutilization of surveillance imaging among patients with early-stage testicular cancer in Ontario treated during 2000-2010}

\begin{tabular}{|c|c|c|c|c|c|c|}
\hline & \multicolumn{3}{|c|}{ Years 1-2 } & \multicolumn{3}{|c|}{ Years 3-5 } \\
\hline & \multicolumn{2}{|c|}{ Univariate analysis } & \multirow{2}{*}{$\begin{array}{c}\begin{array}{c}\text { Multivariate } \\
\text { analysis }\end{array} \\
\text { OR (95\% CI) } \\
\end{array}$} & \multicolumn{2}{|c|}{ Univariate analysis } & \multirow{2}{*}{$\begin{array}{c}\text { Multivariate analysis } \\
\text { OR (95\% CI) }\end{array}$} \\
\hline & Rate & OR (95\% CI) & & Rate & OR (95\% CI) & \\
\hline $2000-2010^{\wedge}$ & & $1.01(0.97-1.05)$ & $0.97(0.93-1.02)$ & & $0.96(0.9-1.02)$ & $0.94(0.88-1.01)$ \\
\hline \multicolumn{7}{|l|}{ Age } \\
\hline$<30$ years & 39 & Ref & Ref & 66 & Ref & Ref \\
\hline $30-39$ years & 52 & $1.67(1.27-2.19)$ & $1.14(0.83-1.56)$ & 64 & $0.94(0.66-1.33)$ & $1.46(0.96-2.21)$ \\
\hline $40+$ years & 56 & $1.97(1.5-2.58)$ & $1.1(0.8-1.51)$ & 58 & $0.72(0.51-1.03)$ & $1.08(0.71-1.63)$ \\
\hline \multicolumn{7}{|l|}{ Histology } \\
\hline Non-sem & 26 & $0.22(0.17-0.29)$ & $0.24(0.18-0.33)$ & 78 & $3.05(2.19-4.25)$ & $4.01(2.58-6.24)$ \\
\hline Seminoma & 61 & Ref & Ref & 54 & Ref & Ref \\
\hline \multicolumn{7}{|l|}{ Risk $^{*}$} \\
\hline High & 54 & Ref & Ref & 55 & Ref & Ref \\
\hline Low & 49 & $0.8(0.64-1.01)$ & $1.02(0.79-1.31)$ & 67 & $1.67(1.23-2.27)$ & $1.38(0.99-1.92)$ \\
\hline
\end{tabular}

Per year. "High-risk disease is defined as: LVI for NSGCT; tumour $>4 \mathrm{~cm}$ and/or rete testis involvement for seminoma. Geographic region was included in the multivariate model and was significantly associated with imaging over-utilization both in years 1-2 and years 3-5 (data not shown). CI: confidence interval; OR: odds ratio. 